\title{
Mutant ubiquitin found in Alzheimer's disease causes neuritic beading of mitochondria in association with neuronal degeneration
}

\author{
Z Tan ${ }^{\star, 1,2}$, X Sun ${ }^{3}$, F-S Hou ${ }^{4}$, H-W Oh ${ }^{3}$, LGW Hilgenberg ${ }^{3}$, EM Hol ${ }^{5}$, FW van Leeuwen ${ }^{6}$, MA Smith ${ }^{3}$, DK O'Dowd ${ }^{3}$ and SS Schreiber ${ }^{1,2,3,7}$
}

A dinucleotide deletion in human ubiquitin (Ub) B messenger RNA leads to formation of polyubiquitin (UbB) +1 , which has been implicated in neuronal cell death in Alzheimer's and other neurodegenerative diseases. Previous studies demonstrate that $\mathrm{UbB}+1$ protein causes proteasome dysfunction. However, the molecular mechanism of UbB +1 -mediated neuronal degeneration remains unknown. We now report that $\mathrm{UbB}+1$ causes neuritic beading, impairment of mitochondrial movements, mitochondrial stress and neuronal degeneration in primary neurons. Transfection of $\mathrm{UbB}+1$ induced a buildup of mitochondria in neurites and dysregulation of mitochondrial motor proteins, in particular, through detachment of P74, the dynein intermediate chain, from mitochondria and decreased mitochondria-microtubule interactions. Altered distribution of mitochondria was associated with activation of both the mitochondrial stress and p53 cell death pathways. These results support the hypothesis that neuritic clogging of mitochondria by $\mathrm{UbB}+1$ triggers a cascade of events characterized by local activation of mitochondrial stress followed by global cell death. Furthermore, UbB +1 small interfering RNA efficiently blocked expression of UbB +1 protein, attenuated neuritic beading and preserved cellular morphology, suggesting a potential neuroprotective strategy for certain neurodegenerative disorders.

Cell Death and Differentiation (2007) 14, 1721-1732; doi:10.1038/sj.cdd.4402180; published online 15 June 2007

Ubiquitin (Ub) is a highly conserved 76-amino acid protein that facilitates 265 proteasome-mediated proteolysis in cells. Ub covalently tags a wide variety of proteins by the sequential actions of an E1-activating enzyme, an E2conjugating enzyme that transfers Ub to a target protein, and an E3 ligase that ubiquitinates the target protein. ${ }^{1}$ Polyubiquitinated proteins are then degraded through the $26 \mathrm{~S}$ proteasome complex. To generate an efficient proteasome-targeting signal, $\mathrm{Ub}$ is first linked to a given substrate by formation of an isopeptide bond between the C-terminal glycine of $\mathrm{Ub}(\mathrm{G} 76)$ and an internal lysine $(\mathrm{K})$ residue of the substrate. Polyubiquitination occurs through the formation of K48-G76 isopeptide bonds between successive Ub molecules. The Ub-proteasome pathway (UPP) is the major extralysosomal pathway responsible for protein degradation in eukaryotes. ${ }^{1}$ Converging lines of evidence have shown that a variety of intracellular regulatory molecules as well as misfolded proteins are substrates of the UPP. The UPP thus plays a critical role in vital cellular processes such as the cell cycle and cell division, immune and inflammatory responses, cell differentiation, DNA damage repair and cell death. ${ }^{1,2}$
Abnormal UPP function has been implicated in many pathological conditions, including human neurodegenerative diseases. ${ }^{2,3}$ Insoluble ubiquitinated protein aggregates are present in the pathological hallmarks of Alzheimer's disease (AD), particularly neuritic plaques and neurofibrillary tangles. Failure of UPP function has been linked to beta-amyloid $(\mathrm{A} \beta)$ toxicity in the central nervous system (CNS). ${ }^{4}$ UPP dysfunction is also caused by the Ub mutant, $\mathrm{UbB}+1$, which accumulates in $\mathrm{AD}$ and other tauopathies. ${ }^{5-7} \mathrm{UbB}+1$ is generated by a non-DNA-encoded dinucleotide deletion in the first coding unit of human polyubiquitin $B(\mathrm{UbB})$ transcripts. This leads to $a+1$ frameshift mutation resulting in replacement of the C-terminal G76 of Ub with a 20-residue extension. ${ }^{7}$ The altered C-terminus abrogates the ability of $\mathrm{UbB}+1$ to tag other substrates without affecting its polyubiquitination. ${ }^{8,9}$ Polyubiquitinated $\mathrm{UbB}+1$ is refractory to deubiquitination and impairs proteasome function. ${ }^{8}$ Accordingly, induction of $\mathrm{UbB}+1$ results in UPP dysfunction and neuronal cell death. ${ }^{9,10}$ However, the underlying molecular basis of $\mathrm{UbB}+1$ formation and its role in neuronal degeneration remain unclear. Here, we demonstrate the novel finding that $\mathrm{UbB}+1$ causes clogging of mitochondria with beading in

\footnotetext{
${ }^{1}$ Department of Neurology, University of California, Irvine, CA, USA; ${ }^{2}$ Institute for Brain Aging and Dementia, School of Medicine, University of California, Irvine, CA, USA; ${ }^{3}$ Department of Anatomy and Neurobiology, University of California, Irvine, CA, USA; ${ }^{4}$ Optical Biology Core Facility, University of California, Irvine, CA, USA; ${ }^{5}$ Netherlands Institute for Neuroscience, Amsterdam, The Netherlands; ${ }^{6}$ Department of Cellular Neuroscience, University Maastricht, Maastricht, The Netherlands and ${ }^{7}$ VA Long Beach Healthcare System, Long Beach, CA, USA

*Corresponding author: Z Tan, Department of Neurology, University of California Irvine School of Medicine, ZOT 4275, 100 Irvine Hall, Irvine, CA 92697-4275, USA. Tel: + 1949824-1669; Fax: + 1 949824-2436; E-mail: tanz@uci.edu

Keywords: ubiquitin; Alzheimer's disease; mitochondria; axonal transport; neurite; neuronal cell death

Abbreviations: Ub, ubiquitin; UbB +1 , ubiquitin $B+1$; $A D$, Alzheimer's disease; UPP, uibiquitin-proteasome pathway; siRNA, small interfering RNA; $A \beta$, betaamyloid; CNS, central nervous system; MT, microtubule; EGFP, enhanced green fluorescent protein; EYFP, enhanced yellow fluorescent protein; ER, endoplasmic reticulum; TMRM, tetramethyl rhodamine methyl ester; MSR, MitoSox Red; TUNEL, terminal dUTP nick-end labeling; shRNA, small hairpin RNA; RFP, red fluorescent protein; GAPDH, glyceraldehyde-3-phosphate dehydrogenase; EM, electron microscopy; PCR, DNA polymerase chain reaction

Received 14.11.06; revised 20.4.07; accepted 11.5.07; Edited by SA Lipton; published online 15.6.07
} 
neurites, followed by mitochondrial stress and neuronal cell death. Biochemical and ultrastructural analyses suggest that neuritic beading might result from dysregulation of mitochondrial transport proteins and impaired mitochondria-microtubule (MT) interactions. In addition, small interfering RNA (siRNA)-mediated suppression of $\mathrm{UbB}+1$ attenuates the development of neuritic beading and preserves neuronal morphology. Mitochondrial stress induced by a mutant form of $\mathrm{Ub}$ may therefore be a novel mechanism contributing to neuronal loss in $A D$ and other neurodegenerative diseases.

\section{Results}

UbB +1 causes neuritic beading and mitochondrial buildup in neuronal processes. To investigate the pathophysiological effects of $\mathrm{UbB}+1$, primary neurons were transfected with plasmids expressing either enhanced green fluorescent protein (EGFP)-UbB +1 or EGFP-Ub fusion proteins, or EGFP alone. Fluorescence microscopy revealed a pattern consistent with neuritic beading in the processes of most cells by $16 \mathrm{~h}$ after EGFP-UbB +1 transfection (Figure 1a, panel 1). The majority of cells transfected with either EGFP-Ub or EGFP showed normal morphology without development of neuritic beading (Figure 1a, panels 2 and 3 in upper row). Compared to EGFP-alone, cells transfected with EGFP-Ub exhibited a heterogeneous pattern of fluorescence consistent with the non-uniform distribution of endogenous $\mathrm{Ub}$ and Ub-conjugates. ${ }^{11}$ Quantification of the results revealed a statistically significant increase in the number of cells containing neuritic beads with time as well as the number of beads per beading cell following pEGFP-UbB +1 transfection compared with controls (Figure 1a, bottom row). There were no significant differences between results obtained in rat and mouse neurons (data not shown).

As neuritic beading has been associated with jamming of intracellular organelles, ${ }^{12}$ we next examined the nature of the neuritic beads by co-transfecting primary neurons with pEGFP-UbB + 1 and either a mitochondrial (pDsRed2-Mito), endoplasmic reticulum (pEYFP-ER) or Golgi (pEYFP-Golgi)targeting reporter. Analysis by confocal laser optical dissection revealed that almost all of the EGFP-UbB + 1-positive beads contained mitochondria (Figure 1b), whereas endoplasmic reticulum and Golgi bodies were absent (data not shown). To further examine the structure and composition of the neuritic beads, neurons were analyzed by transmission electron microscopy $16 \mathrm{~h}$ after $\mathrm{pEGFP-UbB}+1$ transfection. Consistent with our previous observations mitochondria, or mitochondria-like structures, were the primary components in beads. In addition, the beads contained MTs, vesicular bodies and smaller structures that were possibly aggresomes and other organelles (Figure 1c-e).

Neuritic beading is correlated with mitochondrial motor
dysregulation and altered mitochondria-MT interactions. Mitochondria are bidirectionally transported between the neuronal soma and terminals by the axoplasmic transport machinery. ${ }^{13,14}$ As the accumulation of mitochondria in neuritic beads could potentially result from impaired mitochondrial transport, we evaluated the effects of $\mathrm{UbB}+1$ on mitochondrial movements in neurites and expression of mitochondrial molecular motors. Neurons were co-transfected with the mitochondrial reporter, pDsRed2-Mito and either pEGFP, pEGFP-Ub or pEGFP$\mathrm{UbB}+1$, and the movements of labeled aggregates containing mitochondria were analyzed by time-lapse photomicrography (Figure 2a). Quantification of the results revealed a striking decrease in the number of mitochondriacontaining aggregates that moved retrogradely in EGFP$\mathrm{UbB}+1$ - compared with either EGFP-Ub- or EGFP-expressing cells (Figure $2 \mathrm{~b}$ ). In contrast, there was no significantly statistical difference in the anterograde movement of aggregates from EGFP-UbB +1-expressing cells compared with control EGFP-Ub- or EGFP-expressing cells (Figure 2b).

Mitochondria are transported as cargo along neuronal processes through direct interactions between the motor molecules, KIF1B kinesin and dynein, and the MT cytoskeletal system. ${ }^{15,16}$ Therefore, we examined the overall expression levels of KIF1B, a member of the kinesin superfamily that is a major motor for anterograde transport of mitochondria, ${ }^{15}$ and dynein intermediate chain $\mathrm{P} 74$, which regulates mitochondrial retrograde transport, in transfected neurons. Western blot analyses using a panel of antibodies revealed that EGFP, EGFP-Ub and EGFP-UbB +1 expression increased between 8 and $30 \mathrm{~h}$ post-transfection (Figure $3 \mathrm{a}$, row 1 ). $\mathrm{UbB}+1$ immunoreactivity was confirmed with a specific antibody against $\mathrm{UbB}+1$ (Figure $3 a$, row 2). Whereas there was no statistically significant change in the abundance of P74 in whole-cell lysates, a significant decrease in mitochondriabound P74 was detected $16 \mathrm{~h}$ after $\mathrm{UbB}+1$ transfection, along with a corresponding increase in cytosolic dynein (Figure 3a, rows 4, 6 and 9; Figure 3b). Both whole-cell and mitochondria-associated levels of KIF1B remained unchanged (Figure $3 a$, rows $3 \& 7$ ). There were no changes in motor protein expression in cells transfected with either EGFP-Ub or EGFP alone.

To rule out any possible effects of EGFP in the fusion proteins, primary neurons were co-transfected with

Figure $1 \mathrm{UbB}+1$ causes neuritic beading and mitochondrial buildup in neuronal processes. (a) Fluorescence images of rat cortical neurons $16 \mathrm{~h}$ after transfection with pEGFP-UbB +1 , pEGFP-Ub or pEGFP alone. Quantification shows a significant increase in the percentage of beading cells and the number of beads per cell following transfection with pEGFP-UbB $+1 .{ }^{*}$ and ${ }^{\Delta}$ indicate significant differences $\left(P^{\prime}{ }^{*}<0.05,{ }^{* *}<0.01, N=4 ;{ }^{\Delta}<0.05, N=20\right.$, as per ANOVA and Fisher's PLSD). Bars depict mean \pm S.E. (b) Neurons were co-transfected with pEGFP-UbB +1 and pDsRed2-Mito. Confocal microscopy shows colocalization of the mitochondrial marker in EGFP$\mathrm{UbB}+1$-associated neuritic beads. (c) A transmission electron micrograph reveals beading structures in an axon from a pEGFP-UbB +1 -transfected cell. (d and e) At higher magnification, the boxed areas in (c) show the presence of microtubules (MT), mitochondria or mitochondria-like structures (Mi), spheroids (broad arrows in e) and smaller vesicular bodies. (f) A neuritic bead containing mitochondria in clumps, MT and vesicular bodies. An adjacent process is demarcated by delimiting membranes (DM, arrowheads). Scale bar $=50 \mu \mathrm{m}$ (a), $20 \mu \mathrm{m}$ (insets a), $150 \mu \mathrm{m}$ (inset b) shown at higher magnification in other panels (30 $\mu \mathrm{m}), 1 \mu \mathrm{m}$ (c) and $0.4 \mu \mathrm{m}$ in (d-f) 
a
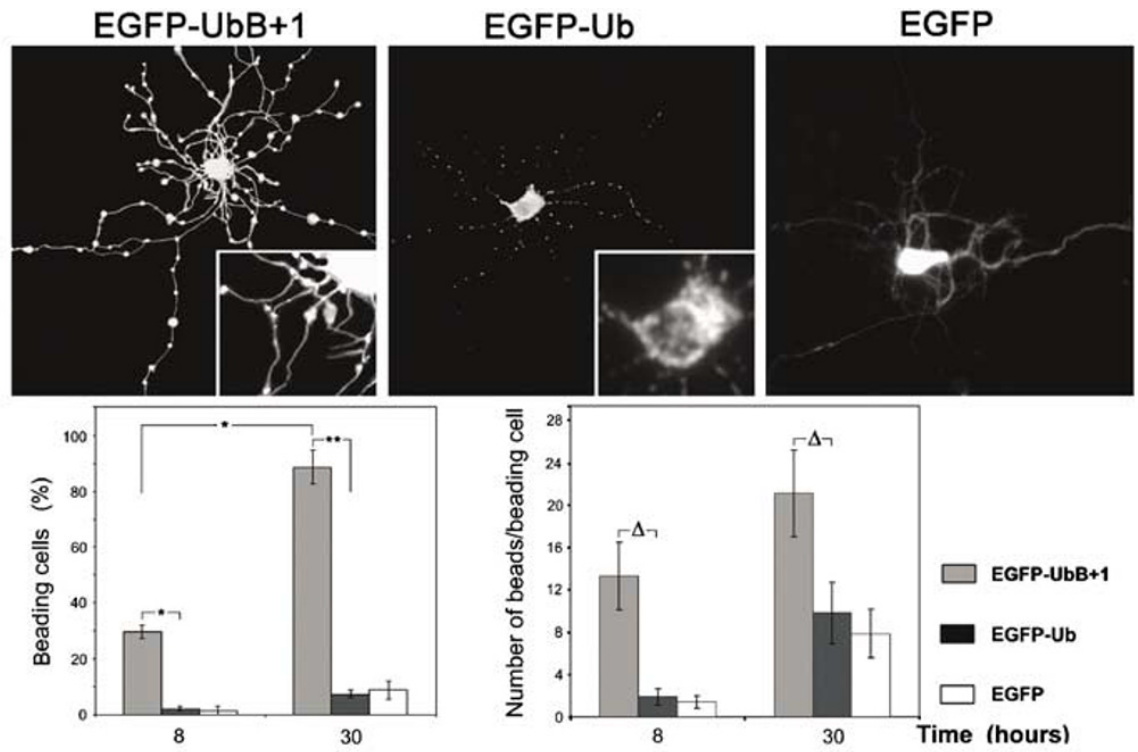

b

EGFP-UbB+1

DsRed2-Mito

Colocalization
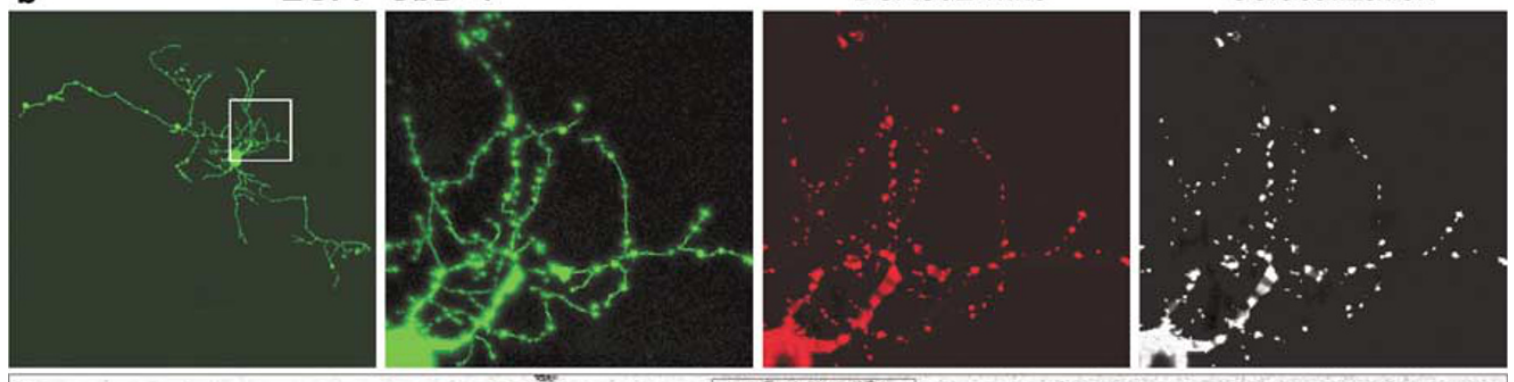

C

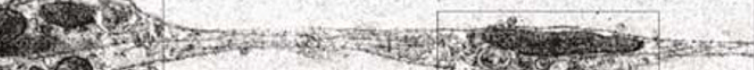
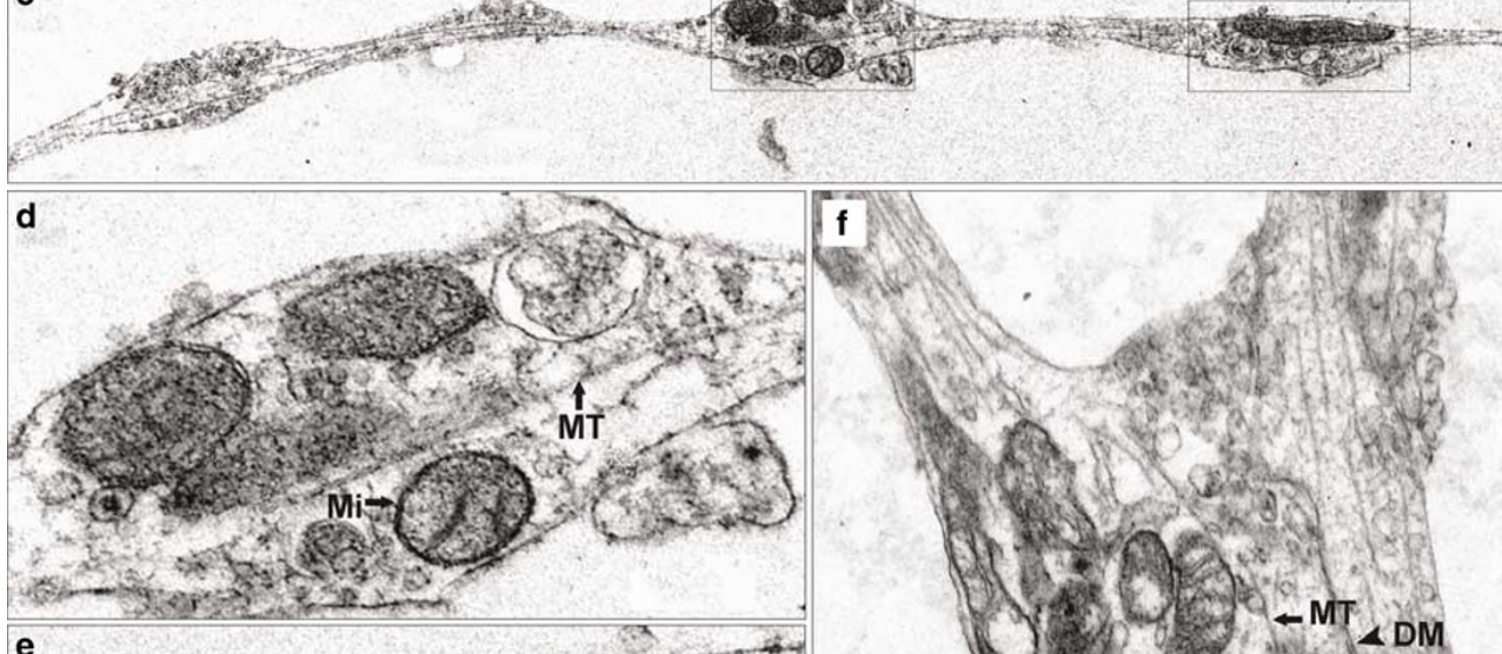

e

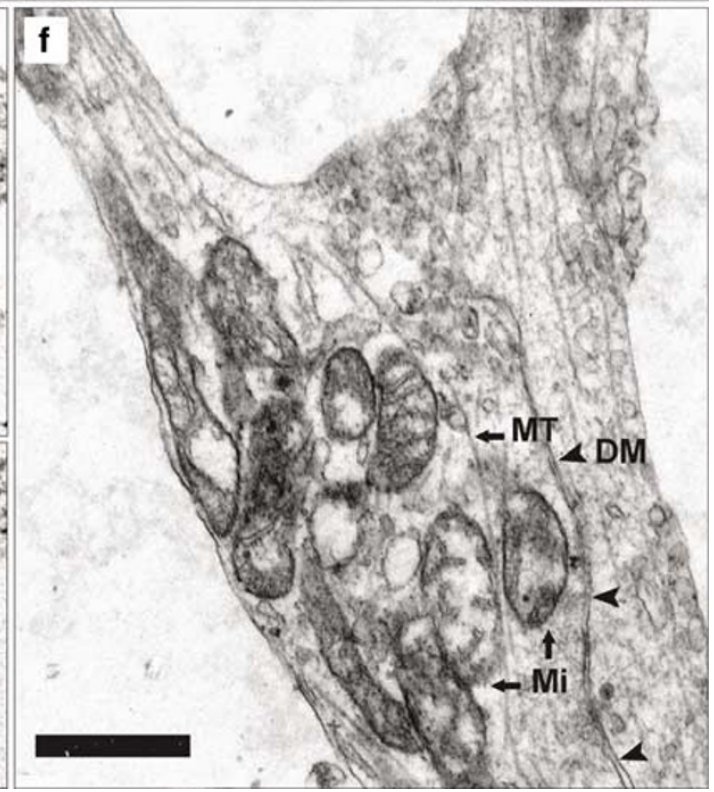



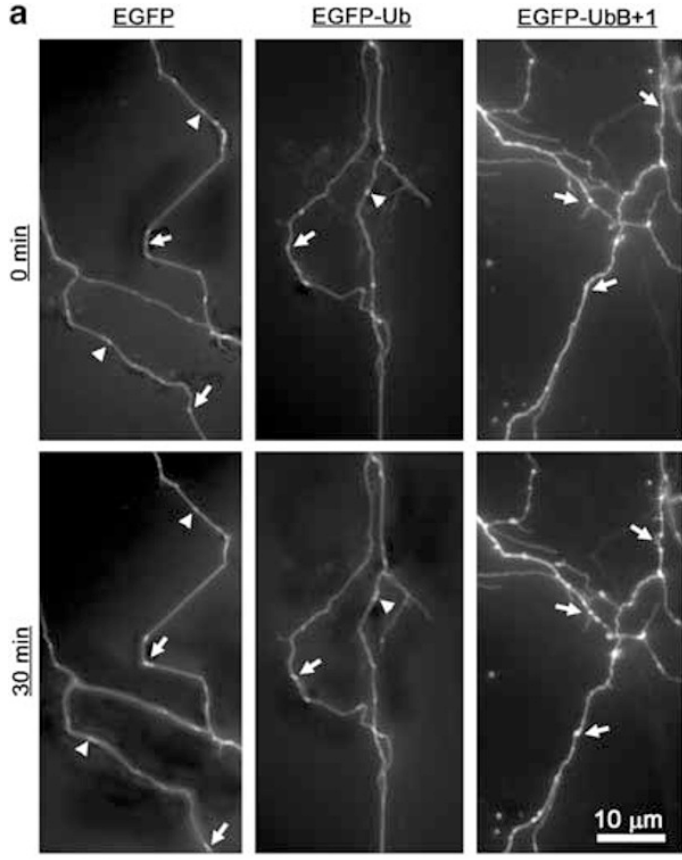

b

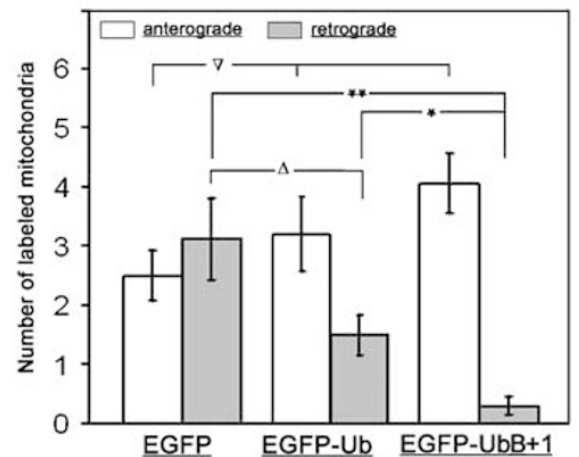

Figure 2 Time-lapse analysis of mitochondrial movements. (a) Comparison of photomicrographs at two different times after transfection demonstrates retrograde (arrowheads shifted up) and anterograde (arrows shifted down) motions of mitochondria-containing aggregates. Scale bar $=10 \mu \mathrm{m}$. (b) Quantification of mitochondrial aggregate motions in neuronal processes. The number of labeled mitochondrial aggregates that moved retrogradely was significantly reduced in EGFP-UbB + 1-transfected cells ( $P^{\prime} s^{*}<0.01,{ }^{* *}<0.005, N=25$, as per ANOVA and Fisher's PLSD). No significant difference was detected between the number of mitochondrial aggregates that moved retrogradely in cells expressing EGFP-Ub and Ub alone ( $P$ 's $\Delta>0.45, N=25$, as per ANOVA); no statistically significant change was detected in the number of mitochondrial aggregates that moved anterogradely in EGFP-UbB + 1-expressing cells or in cells expressing either EGFP-Ub or EGFP alone ( $P S \nabla>0.50, N=25$, as per ANOVA). Bars depict mean \pm S.E.

DsRed2-Mito and a pcDNA3.1 plasmid expressing nontagged $\mathrm{Ub}$ or $\mathrm{UbB}+1$. Dual fluorescence of DsRed2-Mito and $\mathrm{UbB}+1$ immunoreactivity revealed similar beading pattern of mitochondria in neurites in cells expressing $\mathrm{UbB}+1$ (Figure 3c). Western blots also demonstrated a similar decrease in abundance of mitochondria-associated P74 with a corresponding increase in the level of cytoplasmic P74 in neurons transfected with $\mathrm{UbB}+1$, whereas empty vector and Ub controls showed no change (Figure 3d).

As mitochondrial transport is facilitated by direct interactions with molecular motors, we investigated whether $\mathrm{UbB}+1$ protein interfered with binding of P74 to mitochondria cargo. In a cell-free reconstitution assay, isolated mitochondrial fractions from untreated neurons were incubated with wildtype $\mathrm{Ub}, \mathrm{UbB}+1$ or $\mathrm{Ub} 5+1$, that is, $\mathrm{UbB}+1$ conjugated with a tetra-polyUb chain through K48. Increasing amounts of $\mathrm{UbB}+1$ or $\mathrm{Ub} 5+1$ resulted in a significant decrease in mitochondria-associated P74 and a corresponding increase in the buffer fraction (Figure 3e). These results indicate that both free and polyubiquitinated forms of $\mathrm{UbB}+1$ protein may interfere with P74 binding to mitochondria and result in detachment of P74 from its cargo.

Although mechanisms of mitochondrial transport are not well understood, studies have revealed that motors actively move their cargo along MTs. ${ }^{17}$ We speculated that detachment of P74 from mitochondria may result in the dissociation of mitochondria from MTs. The effect of $\mathrm{UbB}+1$ on the spatial relationship between mitochondria and MTs was investigated by an ultrastructural analysis of longitudinal sections through neurites. In EGFP- or EGFP-Ub-transfected neurons, the majority of mitochondria were flanked by MTs on two sides, that is, 'MT-associated'. In contrast, most of the mitochondria in EGFP-UbB + 1-transfected neurons were either not adjacent to or contiguous with MTs on only one side. Quantification of these results demonstrated a dramatic reduction of MT-associated mitochondria in EGFP-UbB + 1-transfected cells (Figures 4a, b).

Neuritic beading is associated with mitochondrial stress. To explore the downstream effects of $\mathrm{UbB}+1$ expression in more detail, several indicators of mitochondrial stress were employed. The loss of mitochondrial membrane potential was evaluated by staining with tetramethyl rhodamine methyl ester (TMRM) and mitochondrial superoxide production was evaluated by MitoSOX-Red (MSR). TMRM was observed to stain almost all the mitochondria in EGFP-transfected cells (data not shown) and EGFP-Ub-transfected cells (Figure $5 a$, second row). In contrast, cells expressing EGFP-UbB +1 exhibited reduced TMRM staining in both neuronal processes and the cell somata (Figure $5 a$, first row). Specifically, reduced TMRM fluorescence in neurites preceded the more global loss of TMRM fluorescence over the entire cell (Figure 5b). Consistent with the loss of mitochondrial membrane potential, MSR signal colocalized with EGFP-UbB +1 in neuritic beads (Figure $5 \mathrm{c}$ ). Cells expressing EGFP-Ub were MSRnegative (Figure $5 \mathrm{c}$ ). Quantification of these results demonstrated a progressive increase in the number of MSR-positive cells with time after EGFP-UbB + 1-transfection (Figure $5 \mathrm{~d}$ ). Importantly, the loss of mitochondrial membrane potential was associated with the release of cytochrome $c$ into the cytosol (Figure 5e). Local elevations in cytochrome $c$ might then act as a trigger for caspase-3 activation in the vicinity of $\mathrm{UbB}+1$-positive neuritic beads (Figure 5f).

$\mathrm{UbB}+1$-induced proteasomal dysfunction is associated with activation of p53 and neuronal cell death. Although our results suggested that adverse effects on mitochondrial movements play a critical role in UbB +1 -mediated neuronal death, we were interested in exploring other potential actions 

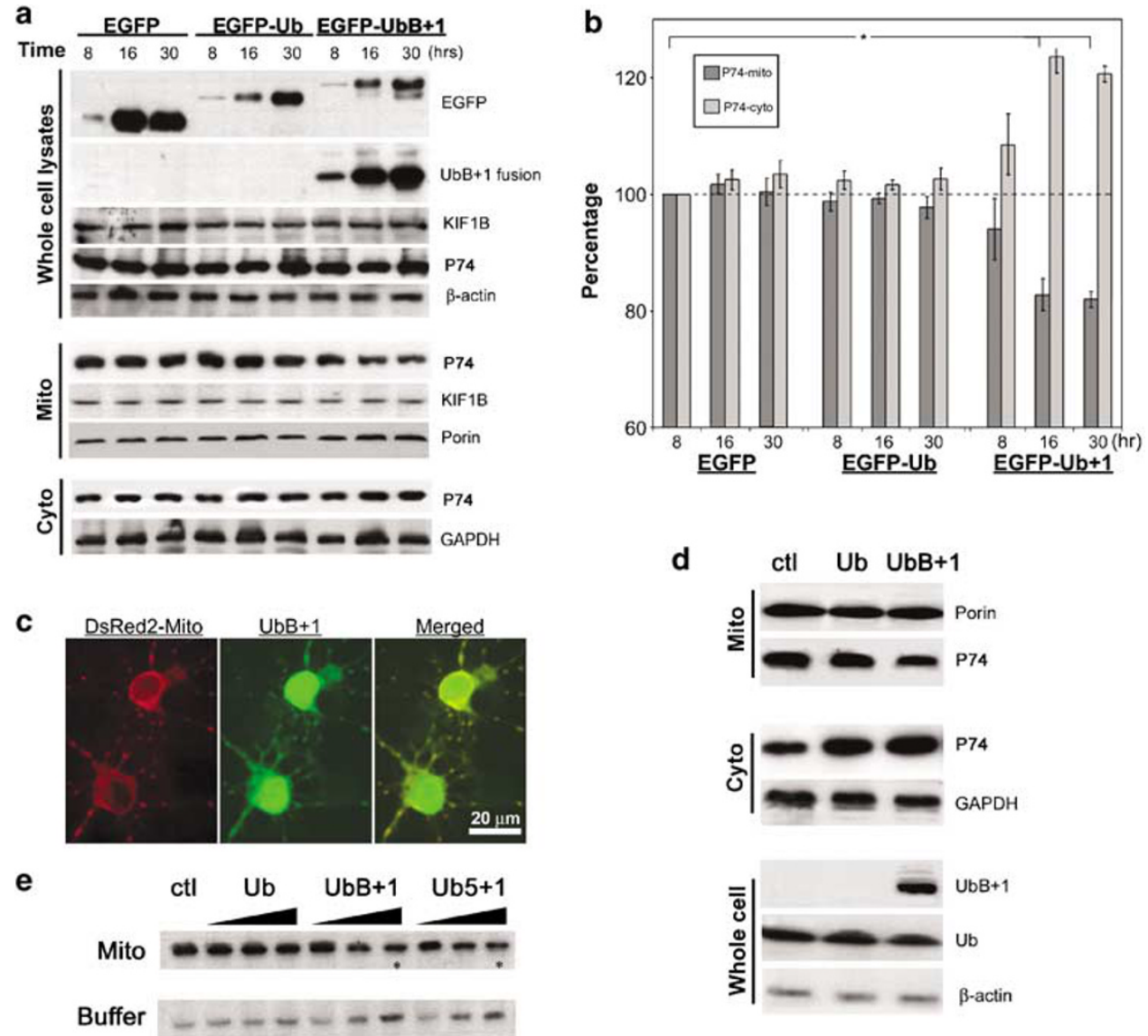

Figure 3 Biochemical analyses of mitochondrial motors. (a) Whole-cell lysates, mitochondrial (mito) and cytosolic (cyto) fractions were prepared from primary neurons transfected with either pEGFP, pEGFP-UbB +1 or pEGFP-Ub. Western blotting was performed with a panel of antibodies at the indicated times. (b) The relative changes in dynein P74 abundance in both mitochondrial and cytosol fractions are shown based on the initial levels in EGFP-transfected cells. Bars represent the mean \pm S.E. from four independent experiments ( ${ }^{\star} P<0.05$, as per Student's $t$-test). (c) Fluorescence microscopy revealed neuritic beading of mitochondria (red) in primary neurons co-transfected with pDsRed2-Mito and UbB + 1 (green) in pcDNA3.1. (d). Whole-cell lysates (whole cell), mitochondrial (mito) and cytosolic (cyto) fractions were prepared from neurons $24 \mathrm{~h}$ after transfection with empty pcDNA3.1 (ctl) or with Ub or UbB $+1 \mathrm{cDNA}$ cassette. Western blotting was performed with indicated antibodies. (e) Cell-free reconstitution assay. Isolated mitochondria were incubated with increasing amounts of Ub species or bovine serum album (ctl), followed by P74 Western blotting. * indicates a significant difference of the group incubated with either $2.0 \mu \mathrm{g} \mathrm{UbB}+1$ or $\mathrm{Ub} 5+1$ relative to the control ( $P$ 's $<0.05, N=4$, as per ANOVA and Fisher's PLSD)

of $\mathrm{UbB}+1$ in neurons. The extended $\mathrm{C}$-terminus resulting from the dinucleotide deletion in $\mathrm{UbB}+1$ messenger RNA leads to impaired UPP activity and protein stabilization. ${ }^{9}$ Therefore, we evaluated UPP function by examining the accumulation of polyUb conjugates in transfected neurons. Western blotting revealed a significant increase in abundance of polyUb conjugates in cells expressing EGFP-UbB + 1 compared with either EGFP or EGFP-Ub (Figure 6a). In addition, a marked accumulation of the cell death protein, p53, a major UPP substrate, was also detected by $16 \mathrm{~h}$ after pEGFP-UbB +1 transfection (Figure $6 \mathrm{~b}$ ). Immunocytochemistry revealed a predominantly nuclear accumulation of p53 in beading neurons (Figure 6c, 1st row). However, we could not identify p53 associated with the mitochondrial fractions (data not shown). We previously showed that p53 accumulates, in part, due to decreased availability of free $\mathrm{Ub} .^{18,19}$ In this regard, free $\mathrm{Ub}$ was unchanged in UbB + 1-transfected cells (Figure 6b), suggesting that proteasome inhibition might contribute to the accumulation of p53 in this model. ${ }^{5}$ As p53 is a major transcriptional regulator of apoptosis, it was important to evaluate the transcriptional activity of stabilized p53. Studies with the p53 reporter, pp53-TA-DsRed2, revealed a significant and progressive increase in p53 transactivity (Figure 6c, 2nd row; Figure 6d) and cell death as detected by TUNEL (Figure 6c, 3rd row; Figure 6d) with time after transfection. In contrast, there were relatively few MSRor TUNEL-positive cells following transfection with either EGFP-Ub or EGFP alone (data not shown). Additionally, the role of $\mathrm{p53}$ in $\mathrm{UbB}+1$-induced neuronal cell death was investigated by transfecting primary neurons from p53-null mice with EGFP-UbB + 1, followed by either MSR or TUNEL staining. Although MSR staining was present (Figure 6c, 4th row), there was a striking decrease in the number of MSR-positive neurons in the absence of p53 (i.e., compare Figure $6 \mathrm{e}(23 \pm 4.0 \%)$ with Figure $5 \mathrm{~d}(83.7 \pm 5.5 \%)$ at $48 \mathrm{~h}, P<0.01)$. Similarly, p53-deficiency resulted in a significant reduction in TUNEL-positive neurons (i.e., 
a EGFP-Ub EGFP-UbB+1

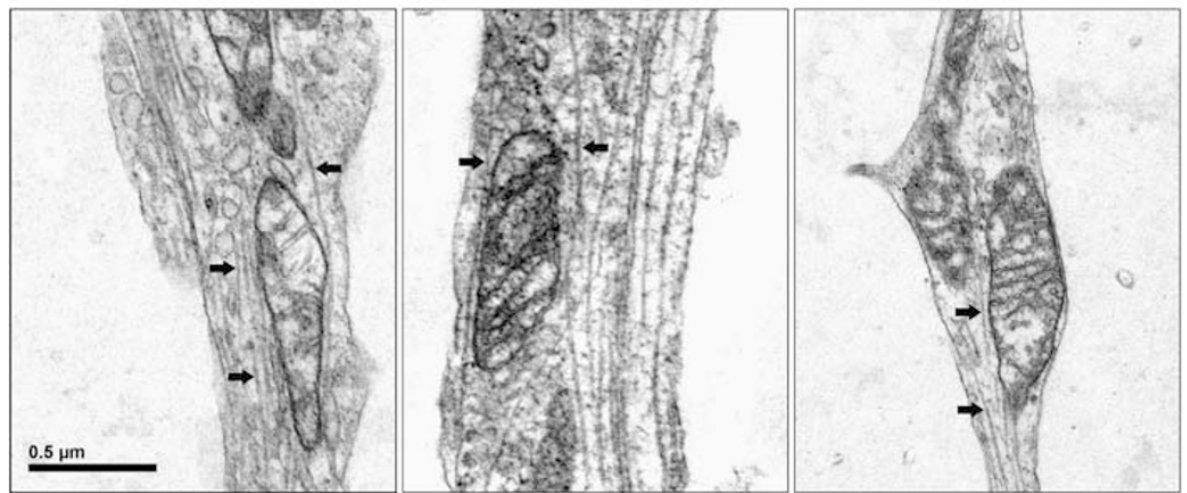

b

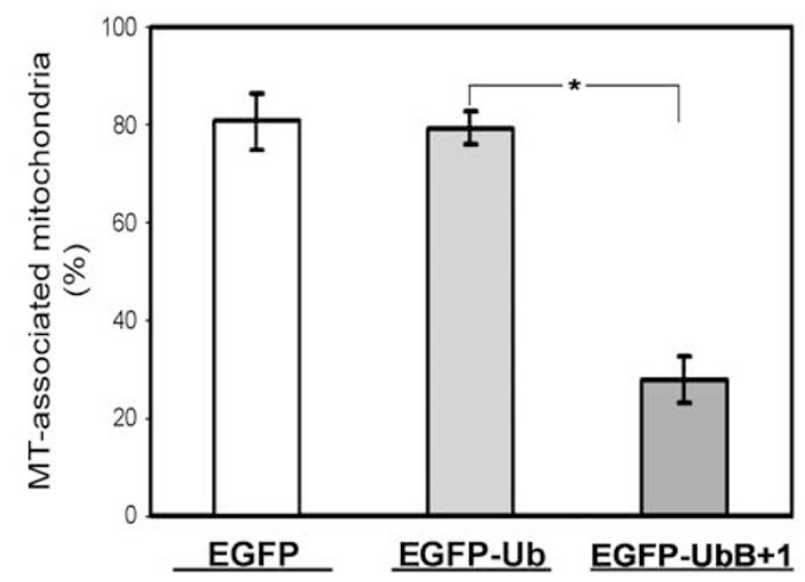

Figure 4 Ultrastructural analysis of the spatial relationships between mitochondria and MTs. (a) Electron micrographs taken from transfected neurons. The first two panels show mitochondria flanked by MTs (arrows) on two sides (i.e., MT-associated), whereas the third panel shows a mitochondrion with MTs on only one side. (b) There was a significant decrease in MT-associated mitochondria relative to the total number of mitochondria-like structures in EGFP-UbB +1 -transfected cells. ( ${ }^{*} P$ ' $<0.01, N=4$, as per ANOVA and Fisher's PLSD). Bars depict mean \pm S.E.

compare Figure 6e $(19.3 \pm 3.9 \%)$ with Figure 6d $(47.3 \pm$ $4.9 \%)$ at $48 \mathrm{~h}, P<0.05)$. To evaluate the importance of caspase-3 activation, primary neurons were transfected with EGFP-UbB +1 with or without the caspase-3-specific inhibitor, DEVD-CHO, followed by TUNEL staining. The results showed that DEVD-CHO significantly attenuated cell death due to EGFP-UbB +1 (Figure $6 \mathrm{~d}$ ).

siRNA-mediated suppression of $\mathrm{UbB}+1$ is neuroprotective. siRNA is a powerful tool that is commonly used for selective suppression of gene expression including allelespecific silencing. ${ }^{20}$ Therefore, we explored the efficacy of siRNA in reversing UbB +1 -mediated toxicity. A sequencespecific UbB +1 siRNA construct was first validated in N2A cells by Western blot analysis (see Supplementary Data). This sequence was then incorporated into a vector coexpressing a short interfering hairpin RNA (shRNA) that targeted $\mathrm{UbB}+1$ (pRFP.UbB $+1 s h$ ) and red fluorescent protein (RFP), DsRed2, to monitor transfection (Figure 7a). An shRNA coding for an irrelevant RNA sequence (pRFP. Controlsh) was used as a control. Studies performed with both N2A cells (see Supplementary Data) and primary neurons showed that pRFP.UbB +1 sh effectively and selec- tively suppressed EGFP-UbB +1 expression (Figure $7 b$, right panels). In contrast, pRFP.Controlsh had no effect on EGFP-UbB +1 expression (Figure $7 \mathrm{~b}$, left panels) and pRFP.UbB +1 sh had no effect on UbB expression (see Supplementary Data). Quantitation revealed that there was a significant decrease in the number of neurons expressing $\mathrm{UbB}+1$ after co-transfection with $\mathrm{UbB}+1$ shRNA compared with the control shRNA (Figure 7c). Importantly, blocking $\mathrm{UbB}+1$ expression in primary neurons prevented the development of neuritic beading and preserved neuronal morphology (Figure 7c, right panels).

\section{Discussion}

Neuritic beading has previously been shown to occur in association with neurodegeneration induced by excitotoxins, $\beta$-amyloid peptide and other neurotoxic agents. ${ }^{21-23}$ Here, we demonstrate that the mutant protein, $\mathrm{UbB}+1$, causes rapid induction of neuritic beading and subsequent cell death. Although protein overexpression may nonspecifically lead to neuritic morphology, changes consistent with neuritic beading were evident only in $\mathrm{UbB}+1$-expressing cells. Further, Western blotting demonstrated that the amounts of 
a

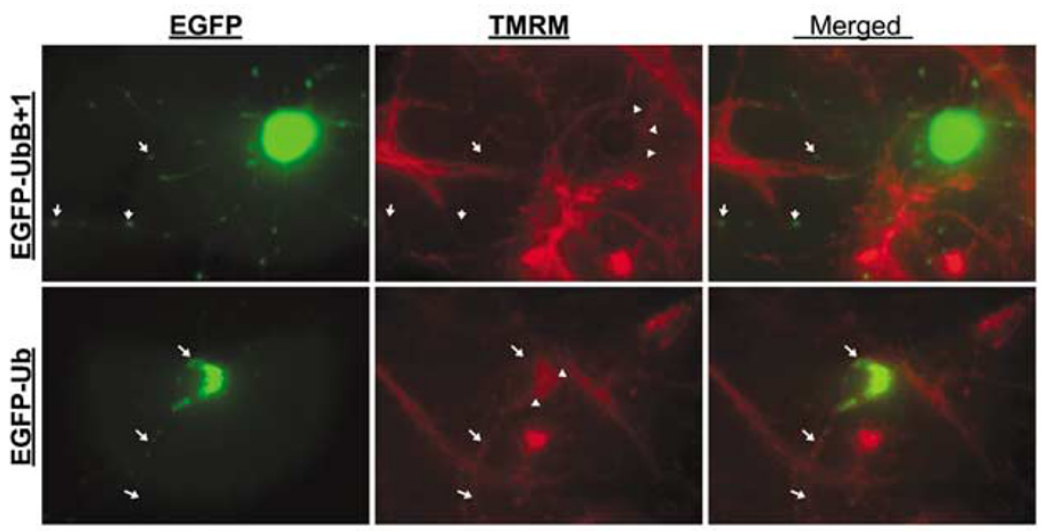

b
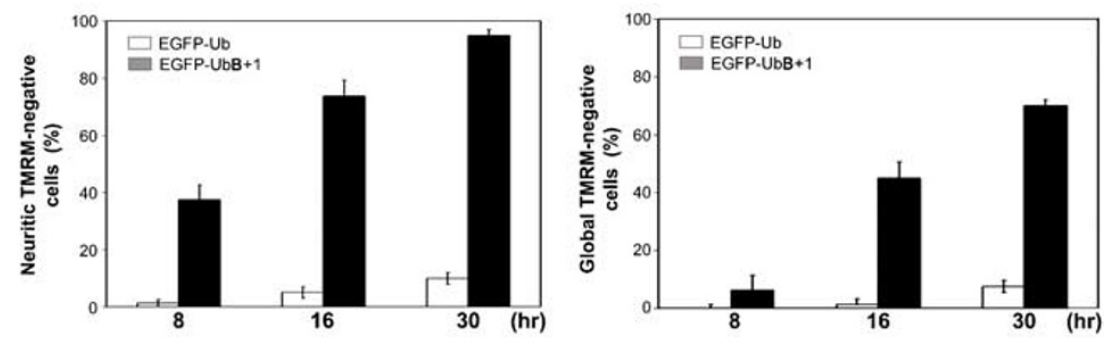

c
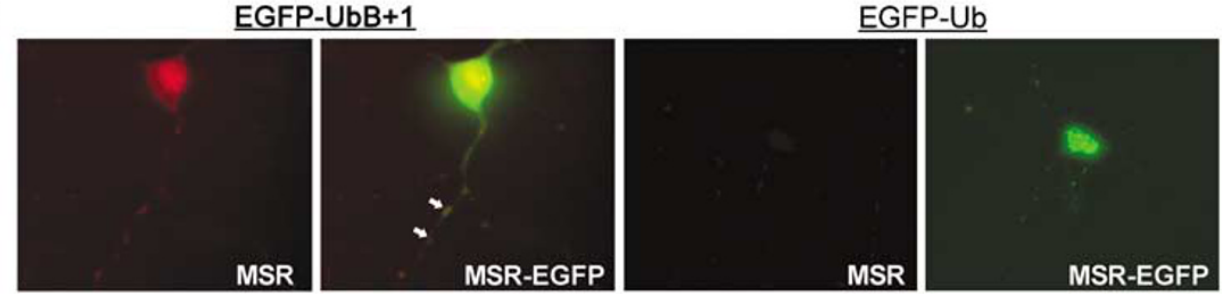

d

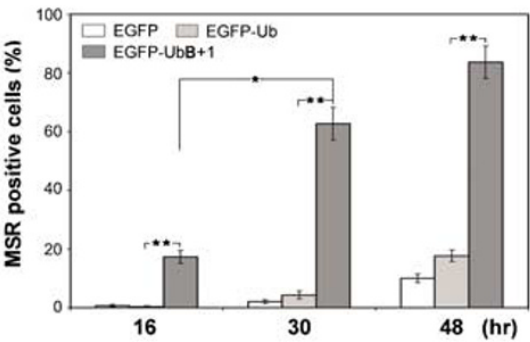

e
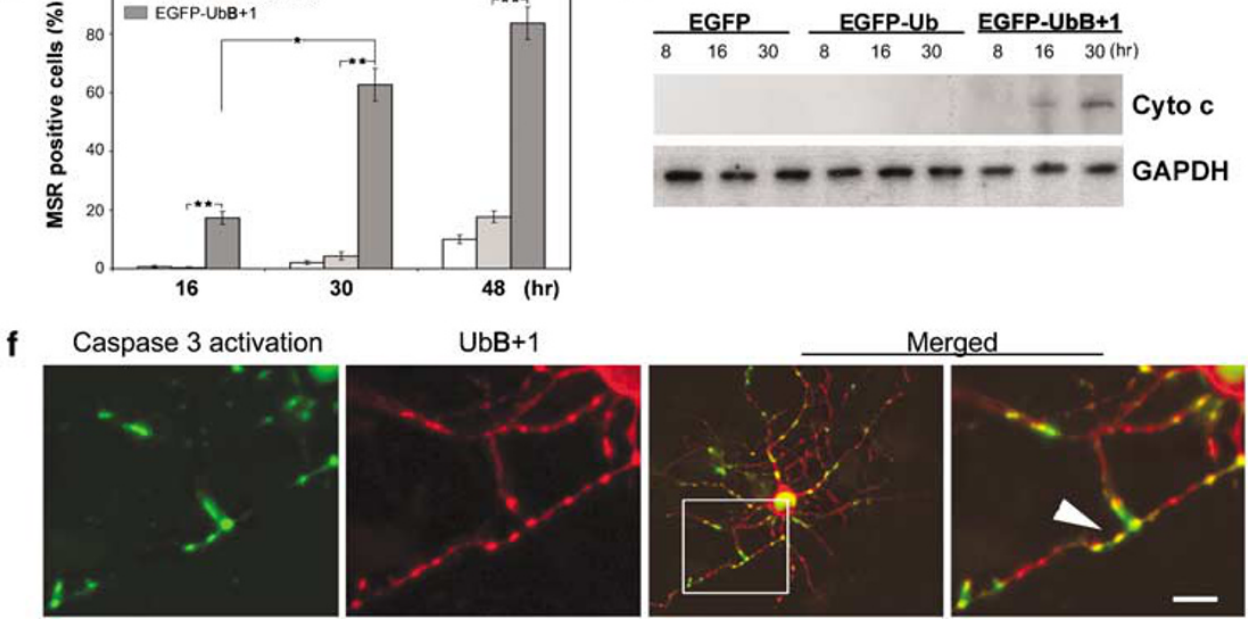

Figure 5 Neuritic beading is associated with mitochondrial stress. (a) Neurons were stained with TMRM after either EGFP-UbB +1 or EGFP-Ub transfection. TMRM fluorescence (red) is present in both neurites (arrows) and cell soma (arrowheads) after EGFP-Ub transfection, but only in the cell soma after EGFP-UbB +1 transfection. (b) The number of cells with reduced TMRM signal in neurites (left) or whole cell (right) relative to EGFP-positive cells following transfection. $P$ 's $<0.01$ for each time point, $N=4$. (c) Mitochondrial superoxides were stained by MSR in EGFP-UbB + 1-associated neuritic beads (arrows), but not in EGFP-Ub-transfected cells. (d) The number of MSRrelative to EGFP-positive cells following plasmid transfection. ${ }^{*}$ Indicates significant differences $\left(P^{\prime} S^{*}<0.05,{ }^{* *}<0.01, N=4\right.$, as per ANOVA and Fisher's PLSD). Bars depict mean \pm S.E. (e) Cytochrome $c$ abundance in cytosol following plasmid transfection. GAPDH was used as loading control. (f) Caspase-3 activation (green) and UbB + 1positive beads (red) are colocalized in some processes (merged, arrow). Scale bar $=40 \mu \mathrm{m}$ (inset $\mathrm{f}$ ), $10 \mu \mathrm{m}$ in other panels of (f), and $15 \mu \mathrm{m}$ in (a and $\mathbf{c}$ )

EGFP-Ub and EGFP-UbB +1 expressed with time after transfection were roughly equivalent. Despite abundant levels of EGFP-Ub on Western blots, we did observe a lower intensity of fluorescence signal in EGFP-Ub-expressing cells (Figure 1a, panel 2). This discrepancy might be due to modulation of fluorescence intensity by interactions between 
a
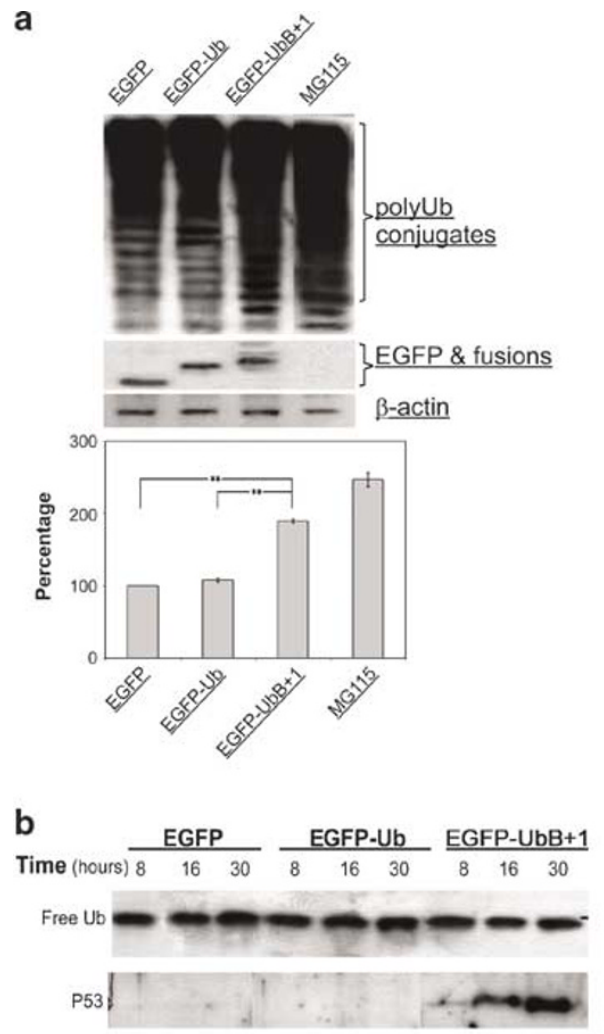

C

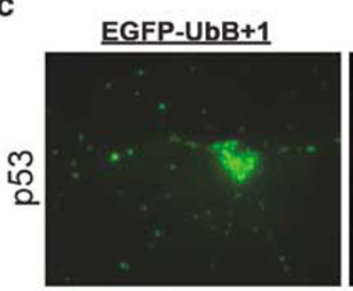

Staining or assay
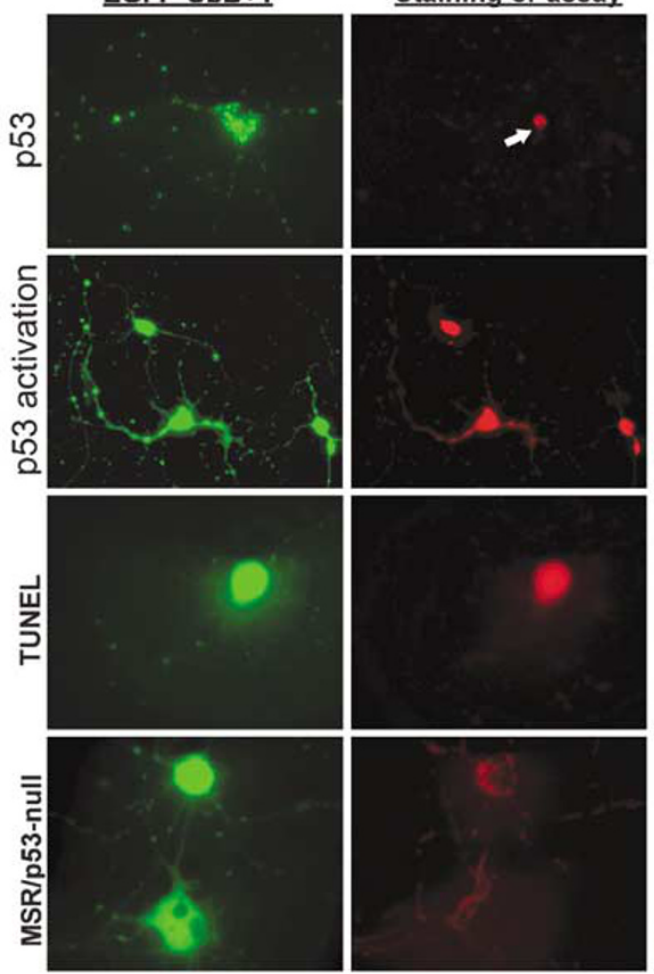
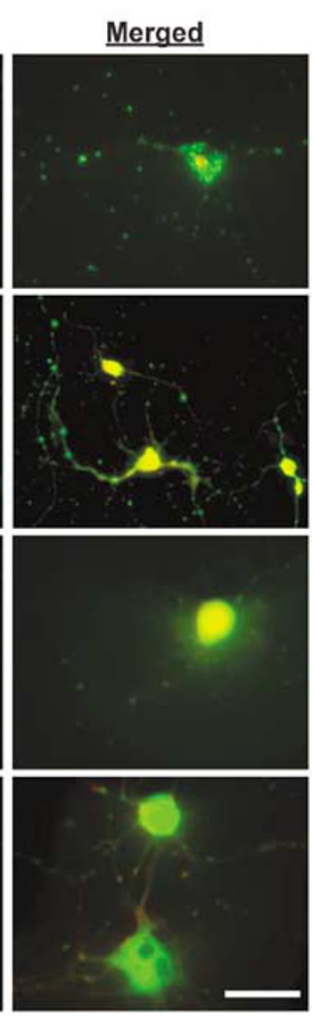
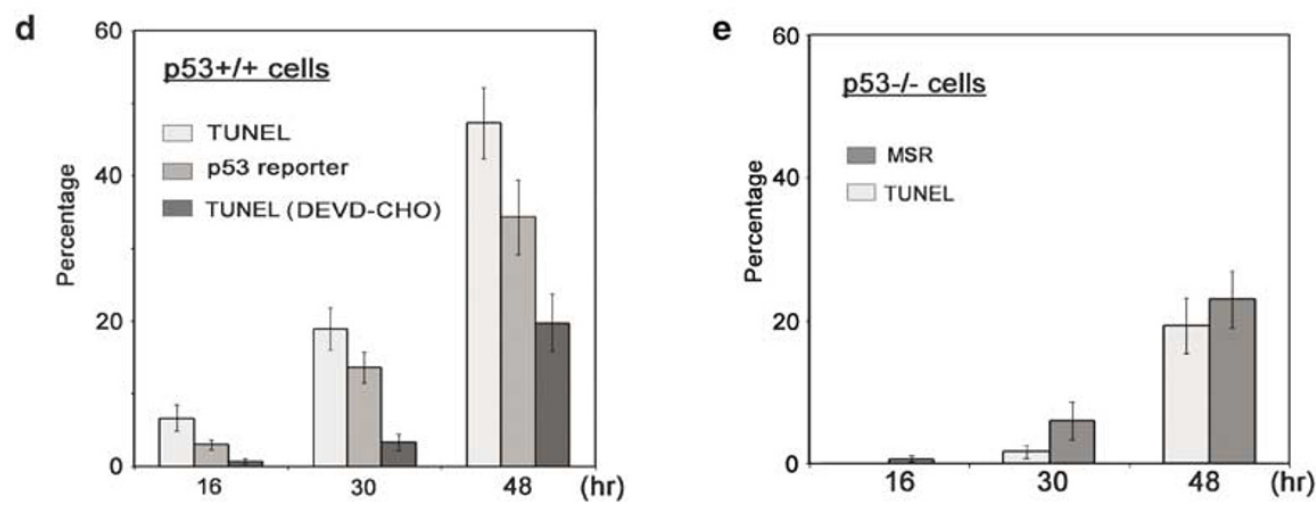

Figure 6 Accumulation of polyUb conjugates, p53 activation and neuronal cell death. (a) Whole-cell lysates were prepared from primary neurons transfected with EGFP or EGFP fusion constructs $24 \mathrm{~h}$ after transfection. Western blotting using a Ub antibody showed increased abundance of polyUb conjugates in UbB +1 -transfected cells. Lysates from neurons treated with MG115 $(5 \mu \mathrm{M}, 24 \mathrm{~h})$ were used as a positive control. Bottom: changes in abundance of total polyUb conjugate ladders are expressed relative to those in EGFP control cells. The data depict the mean number of pixels \pm S.E.M from four independent experiments. (b) p53 and free Ub abundance at indicated times following plasmid transfection. All the lanes shown in each panel were from the same gel. $\beta$-Actin was used as loading control. (c) Representative examples in EGFPUbB + 1-transfected neurons of: nuclear p53 immunoreactivity (arrow, top row); activated p53 following co-transfection with pp53-TA-DsRed2 (second row); positive nuclear TUNEL staining (red, third row) with neuritic beads; positive MSR staining (red, fourth row) in p53-null neurons. Scale bar $=60 \mu \mathrm{m}$ in second row and $30 \mu \mathrm{m}$ in other panels. (d) The number of TUNEL- or 553 reporter-positive cells relative to EGFP-positive cells after EGFP-UbB +1 transfection. Bars are mean $\pm S$.E. TUNEL studies were performed in the presence or absence of DEVD-CHO. (e) p53-null neurons were transfected with EGFP-UbB + 1, and the number of MSR- or TUNEL-positive cells relative to EGFP-positive cells was counted. Bars are mean \pm S.E.

EGFP and UPP-related proteins. ${ }^{24}$ Moreover, neuritic beading of mitochondria was also induced by nontagged $\mathrm{UbB}+1$ (Figure 3c). Taken together, these observations indicate that the demonstrated neuritic effects were specific to $\mathrm{UbB}+1$. A slight increase in the number of beading cells was detected in pEGFP-Ub- or pEGFP-transfected cultures. These changes were not significant and possibly due to minor toxic effects of overexpressed proteins as well as transfection stress.

Ultrastructural studies revealed that the beads contained one or more mitochondria. Although the time frame used for transport measurements in our study was relatively long, significant changes in retrograde movement of mitochondriaassociated aggregates were nonetheless observed, whereas 
a

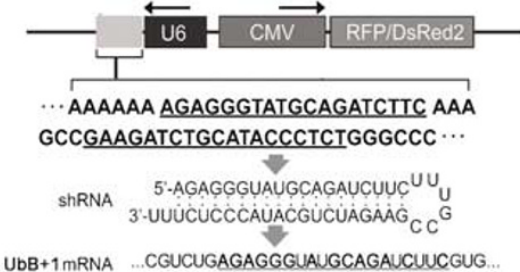

UbB mRNA ...CGUCUGAGAG GUGQUAUGCA GAUCUUGGU.

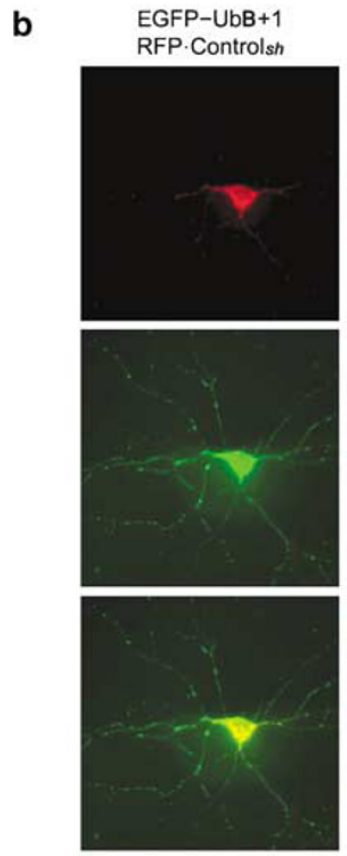

C
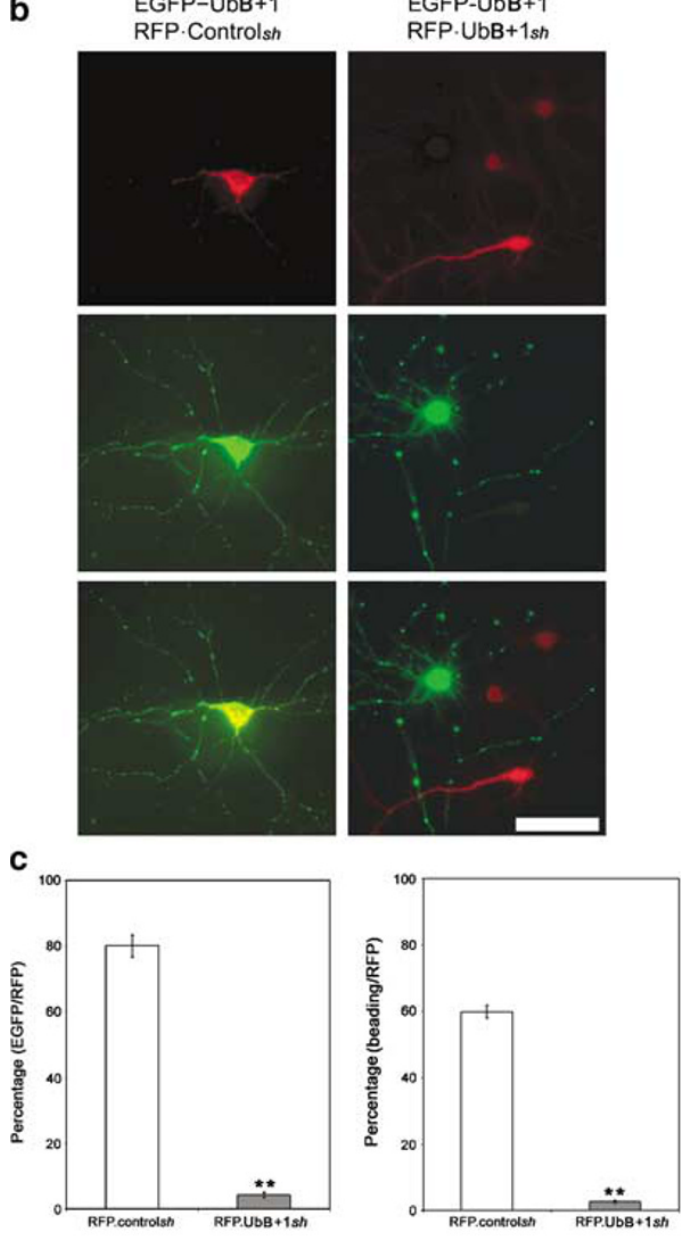

Figure 7 shRNA-mediated suppression of UbB +1 . (a) Schematic of RFP/ DsRed2.U6.silencer vector encoding a specific shRNA directed by murine U6 promoter and a CMV-directed fluorescent reporter. $\mathrm{UbB}+1$ shRNA targets $\mathrm{UbB}+1 \mathrm{mRNA}$, which differs by only two nucleotides (highlighted in red) from UbB mRNA. (b) Mouse cortical neurons were co-transfected with indicated plasmids and visualized by dual fluorescence. Specific suppression of EGFP-UbB +1 by RFP.UbB +1 sh was detected in the cells $24 \mathrm{~h}$ after transfection (right column). Note: one EGFP-UbB + 1-positive cell with neuritic beading in the right lower two panels is RFP.UbB +1 sh-negative. Scale bar represents $30 \mu \mathrm{m}$ for all the panels. (c) The number of EGFP-positive cells (left panel) or cells with neuritic beads (right panel) relative to RFP-positive cells following co-transfection of EGFP-UbB +1 and either RFP.Controlsh or RFP.UbB +1 sh. The bars represent mean \pm S.E. ${ }^{* *} P$ 's $<0.001$

changes in anterograde movement were not significant. More specific analyses of mitochondrial velocities and movements over shorter periods of time may help to delineate the dynamics of mitochondrial movement in injured neurons.
Whereas there was no effect of $\mathrm{UbB}+1$ expression on the overall levels of P74 dynein and KIF1B kinesin, a significant decrease in the abundance of mitochondria-associated P74 was observed. This most likely resulted from detachment of P74 from mitochondrial cargo based on the findings from a cell-free reconstitution assay. The importance of P74 is demonstrated by its role as a key adaptor molecule involved in linking two $530 \mathrm{kDa}$ dynein heavy chains to cargo for retrograde transport. ${ }^{25}$ Accordingly, inhibition of dynein function causes what has been described as 'organelle jams' or 'clogs'. ${ }^{26}$ Our results therefore support the hypothesis that $\mathrm{UbB}+1$-mediated detachment of P74 from mitochondrial cargo disturbs the physiological balance between anterograde and retrograde transport. The finding that mitochondria-MT associations were significantly reduced in the processes of UbB +1 -transfected neurons provides further evidence in support of this hypothesis. There was a minor decrease in retrograde axonal transport that was not statistically significant in EGFP-Ub-transfected cells. This suggests that the overexpression of wild-type Ub might also lead to a slight accumulation of polyUb conjugates, which interferes with motor-MT interactions. The combined effects of $\mathrm{UbB}+1$-induced detachment of P74 from mitochondrial cargo and altered MT associations may profoundly disable dynein-driven retrograde motion, thereby leading to an imbalance between anterograde and retrograde movements and a buildup of mitochondria in neurites. Moreover, both $\mathrm{UbB}+1$ and dynein play key roles in the formation of aggresomes during the cellular response to misfolded proteins. ${ }^{27,28}$ Although we did not specifically identify aggresomes in this study, it is possible that aggresomes may compete with mitochondria for P74 binding and thereby facilitate mitochondrial detachment and impairment of retrograde transport. ${ }^{28} \mathrm{UbB}+1$-mediated actions may also be modulated by heat-shock protein family members, ${ }^{29}$ which may be important regulators of axonal transport. ${ }^{30}$

In addition to mitochondria, the transport machinery conveys other membranous and nonmembranous cargo between the neuronal soma and its processes. ${ }^{17}$ Although we did not detect either ER or Golgi bodies in neuritic beads, the distribution of these organelles and other cargo in neuronal processes might still be perturbed by $\mathrm{UbB}+1$ mediated disruption of axonal transport. Thus, impaired axonal transport with abnormal organelle clustering in neurites has been implicated in the pathophysiology of several neurodegenerative diseases. ${ }^{31}$ Moreover, induction of neuritic beading by impairment of the UPP suggests that the adverse effects of $\mathrm{UbB}+1$ on axonal transport may result, in part, from inhibition of proteasome function. In this regard, proteasome inhibition induces neuritic beading in cultured neurons (Tan et al., unpublished observation), and UbB +1 as well as polyubiquitinated protein aggregates accumulate in the pathological hallmarks of $A D$, such as neurofibrillary tangles and neuritic plaques. ${ }^{7}$ In addition, axon damage has been implicated in the initial stages of Alzheimer's-related neuronal degeneration. ${ }^{32,33}$ Although amyloid precursor protein and presenilin mutations, as well as tau isoforms, may disrupt axonal transport, ${ }^{34,35} \mathrm{UbB}+1$-mediated clogging of mitochondria in neurites may also contribute to the mechanism of axon injury and neuropathology in AD. 
The pathophysiological significance of neuritic beading is underscored by the detection of mitochondrial stress in this study. The results suggest that there is a temporal relationship between activation of mitochondrial stress in neurites and neuronal cell death. Notably, mitochondrial stress indicated by the loss of mitochondrial membrane potential seemed to start in peripheral neurites and then spread toward the cell soma. Loss of mitochondrial membrane potential may lead to a local release of cytochrome $c$ in neurites that triggers the mitochondrial cell death cascade through caspase-3 activation. Accordingly, about $70 \%$ of $\mathrm{UbB}+1$-positive cells exhibited a loss of mitochondrial membrane potential in peripheral neurites relatively early on, which preceded a more global loss of mitochondrial membrane potential, mitochondrial oxidative stress and cell death indicated by TUNEL staining. Our findings therefore support the idea that overwhelming mitochondrial stress in neuronal processes, possibly triggered, in part, by impaired transport, may activate a cell death-signaling cascade that is propagated from the periphery to the cell body in a dying-back fashion.

Our observation that UbB +1 induced a significant accumulation of polyUb conjugates is in agreement with prior studies that demonstrated inhibition of proteasome function by UbB $+1 .^{8,9}$ Impaired UPP function likely contributed to the accumulation of tumor suppressor p53, which is a major UPP substrate. ${ }^{36}$ Our results showed that activation of the p53 pathway plays an important role in $\mathrm{UbB}+1$-induced neuronal degeneration, as cell death was substantially decreased in neurons from p53-null mice. In addition, absence of p53 resulted in a significant reduction in the number of cells exhibiting mitochondrial oxidative stress. These findings suggest that p53 might enhance mitochondrial stress either through direct interaction, as has previously been shown, or an indirect mechanism yet to be defined. ${ }^{37} \mathrm{~A}$ link between impaired proteasome activity and mitochondrial oxidative stress has recently been demonstrated. ${ }^{38}$ In the present study, UbB + 1-mediated UPP dysfunction may therefore facilitate mitochondrial superoxide production and contribute to mitochondrial stress.

The use of siRNA is an efficient way to block expression of specific genes, including alleles that differ by only one nucleotide. $^{20}$ Our results demonstrate that siRNA/shRNA can effectively knockdown expression and prevent the toxic effects of $\mathrm{UbB}+1$ protein in cultured neurons. Thus, siRNA might be a potential therapeutic approach to counteract the accumulation of $\mathrm{UbB}+1$ protein in certain neurological disorders. In addition to $\mathrm{UbB}+1 \mathrm{mRNA}$, dinucleotide deletions in other transcripts may increase with aging due to a process of molecular misreading. ${ }^{39}$ RNA interference may therefore be an effective tool to control the expression of abnormal transcripts associated with aging.

In summary, our results demonstrate that $\mathrm{UbB}+1$ causes a buildup of mitochondria in neurites and suggest that this results from dysregulation of motor protein-MT interactions. Impaired transport may then lead to mitochondrial stress and activation of both the mitochondrial and p53 cell death pathways. siRNA-mediated knockdown of UbB +1 expression prevents neuritic beading and may be an effective therapeutic strategy for certain neurodegenerative diseases. Current investigations are aimed at understanding specific interactions between $\mathrm{UbB}+1$ and other polyubiquitinated protein aggregates and axonal transport motors, and relevant effects on mitochondrial movements. These studies may also help to elucidate molecular mechanisms of axon damage in age-related neurodegenerative diseases.

\section{Materials and Methods}

Antibodies, plasmids and reagents. Rabbit polyclonal antibodies directed against EGFP and full length p53, mouse monoclonal antibodies for $P 74$, that is, dynein intermediate chain, goat polyclonal IgG against KIF1B, goat polyclonal $\lg G$ against kinesin heavy chain, $A / G$ plus agarose beads and biotinylated or HRP-conjugated secondary antibodies were obtained from Santa Cruz Biotechnology (Santa Cruz, CA, USA). Mouse monoclonal lgG against $\beta$-actin, rabbit polyclonal IgGs against Ub, porin/VDAC1 and glyceraldehyde-3-phosphate dehydrogenase (GAPDH) were from Sigma-Aldrich (St Louis, MO, USA). The Cytochrome $c$ Releasing Kit was purchased from Biovision (Mountain View, CA, USA). Rabbit polyclonal IgG for $\mathrm{UbB}+1$ as well as recombinant $\mathrm{UbB}+1$ were obtained from R\&D Systems (Minneapolis, MN, USA). Semisynthetic recombinant Ub5 + 1 protein was from BIOMOL International (Plymouth Meeting, PA, USA). Organelle-targeting reporters and CDNA expression constructs including pEGFPC1, pDsRed2-Mito, pEYFP-ER and pEYFP-Golgi were purchased from Clontech (Palo Alto, CA, USA). pEGFP or pCDNA3.1 containing either a single copy of human $\mathrm{Ub}$ or $\mathrm{UbB}+1$ cDNA was subcloned as described. ${ }^{9}$ pEGFP-UbB was subcloned using polymerase chain reaction (PCR) and CDNA from human brain tissue as template. The fluorescent reporter of p53 activation (pp53-TADsRed2) was subcloned from the pp53-TA-Luc vector (Clontech). All of the DNA oligonucleotides used for subcloning were synthesized by Integrated DNA Technologies (Coralville, IA, USA). Cell culture media and Lipofectamine 2000 transfection reagents were obtained from Invitrogen (Carlsbad, CA, USA). CombiMag Transfection Enhancing reagents were purchased from Boca Scientific (Boca Raton, FL, USA). Purified bovine Ub was from Sigma-Aldrich. PCR reagents and related restriction enzymes were purchased from Promega (Madison, WI, USA) and New England Biolabs (Ipswich, MA, USA), respectively. Silencer siRNA construction kit and pSilencer-1.0 U6 vector were obtained from Ambion (Houston, TX, USA). ECL reagents for Western blotting were obtained from Amersham Biosciences (Piscataway, NJ, USA). DEVD-CHO was purchased from Biosource (Camarillo, CA, USA). In Situ Cell Death Detection Kit - TMR Red was obtained from Roche (Indianapolis, IN, USA).

Animals, cell cultures and transfections. Timed-pregnant SpragueDawley rats and C57B//6 mice were purchased from Charles River Laboratories (Wilmington, MA, USA). p53-null (B6.129S2-Trp53 ${ }^{\text {tm1Tyj})}$ breeding pairs were obtained from the Jackson Laboratory (Bar Harbor, ME, USA). All animals were housed in the University of California Irvine School of Medicine Vivarium on a 12-h light/dark cycle with food and water available ad libitum. p53-null genotype was confirmed by PCR according to the vendor's protocol. Primary cortical neurons were prepared from embryonic day 18 (E18) Sprague-Dawley rats and postnatal day 1 (P1) mice and maintained as described previously. ${ }^{18}$ For electron microscopy studies, neurons were grown on poly-D-lysine-coated Eppendorf CELLocate coverslips (Brinkmann Instruments, Westbury, NY, USA). All procedures were approved by the Institutional Animal Care and Use Committee of the University of California Irvine School of Medicine. Mouse N2A neuroblastoma cells were grown in MEM supplemented with heat-inactivated fetal bovine serum (10\%), pyruvate and nonessential amino acids. Plasmid transfections were performed with Lipofectamine 2000 (Invitrogen) in six-well plates following the manufacturer's protocol. Briefly, mixtures of Lipofectamine 2000 diluted in DMEM and plasmid(s) were incubated at room temperature for $20 \mathrm{~min}$ and directly applied to each well with $2 \mathrm{ml}$ fresh serum-free MEM for N2A cells or Neurobasal medium for neurons. Fresh media were substituted $8 \mathrm{~h}$ after transfection. To enhance the transfection efficiency of nontagged $\mathrm{Ub}$ and $\mathrm{UbB}+1$ genes in pcDNA3.1 plasmids, magnetofection was performed using CombiMag transfection kit combined with Lipofectamine 2000 in six-well plates according to the manufacturer's instruction. Transfection efficiencies as determined by both phase contrast and fluorescence microscopy ranged from $70-90 \%$ in N2A cells and $15-30 \%$ in primary neurons.

In situ functional activity assays, immunocytochemistry and TdTmediated dUTP nick end labeling (TUNEL). The transcriptional activity of p53 protein was evaluated by co-transfection of pp53-TA-DsRed2 $(0.4 \mu \mathrm{g} / \mathrm{well})$ 
and either pEGFP-UbB +1 or pEGFP-Ub (1.6 $\mu \mathrm{g} /$ well) as described above. Changes in mitochondrial membrane potential, mitochondrial oxidative stress and caspase-3 activation were assayed using TMRM, MitoSox Red (MSR) (Molecular Probe/Invitrogen) and the CaspaTag Fluorescein Caspase Activity Kit (Intergen, Purchase, NY, USA), respectively, following the manufacturers' protocols. Cells were then fixed with $4 \%$ paraformaldehyde in $1 \times \mathrm{PBS}(\mathrm{pH} 7.4)$ at $4{ }^{\circ} \mathrm{C}$ for $20 \mathrm{~min}$ and immunostained as described previously. ${ }^{18,19}$ Primary antibody against p53 or $\mathrm{UbB}+1$ protein was used at a concentration of $1: 100$. Neuronal cell death was evaluated by TUNEL using an In Situ Cell Death Detection kit following the manufacturer's protocol. In some experiments, TUNEL was evaluated in neurons transfected with EGFP-UbB +1 with or without the caspase-3-specific inhibitor, DEVD-CHO $(20 \mu \mathrm{M})$.

Microscopy and image analysis. Fluorescence microscopy was performed with a Leica DMLB microscope. Images were recorded using a Spot II CCD camera with equal exposure times for specific fluorescence reporters or antibodies. For quantification, all EGFP-positive neurons were counted in 10 randomly chosen fields at a magnification of $\times 200$. Those neurons showing three or more neuritic beads in their processes were counted as 'beading cells'. Up to 60 beading cells were included in the analysis of the number of beads per beading cell. For each of the functional assays, that is, TMRM or MSR staining, p53 activation and caspase-3 activity, results were tabulated for a total of 60 randomly counted EGFP- or UbB + 1-positive cells. Only those EGFP-UbB +1 neurons that showed lack of TMRM staining in at least three distinguishable EGFP-highlighted beads were counted as neuritic TMRM-negative cells. Colocalization studies of neuritic beads and mitochondria were performed with a BioRad confocal laser optical dissection system.

Time-lapse analysis of mitochondrial movements. Neurons were co-transfected with DsRed2-Mito and either EGFP, EGFP-Ub or EGFP-UbB + 1 Movements of mitochondria-containing aggregates were analyzed in neuronal processes from 25 randomly chosen neurons $16-18 \mathrm{~h}$ after transfection. Anterograde and retrograde movements were quantified by a direct comparison of two photomicrographs taken of the same field $30 \mathrm{~min}$ apart. The results are depicted as the average number of labeled mitochondria moving retrograde or anterograde within a given field.

Electron microscopic analysis. Transfected neurons grown on glass coverslips were randomly selected and pre-positioned against an etched grid based on EGFP fluorescence. Cells were then sequentially fixed in $2 \%(w / v)$ paraformaldehyde and $2 \%(\mathrm{v} / \mathrm{v})$ glutaraldehyde in $0.1 \mathrm{M}$ phosphate buffer $(\mathrm{PB}, \mathrm{pH}$ 7.4) at $4^{\circ} \mathrm{C}$ for $1 \mathrm{~h}$, rinsed with $\mathrm{PB}$, postfixed with $1 \% \mathrm{OsO}_{4}$-buffered solution for $1 \mathrm{~h}$, pre-stained with $1 \%(\mathrm{w} / \mathrm{v})$ uranyl acetate for $1 \mathrm{~h}$, dehydrated in ethylic alcohol and embedded in Epon $812\left(50^{\circ} \mathrm{C} \times 24 \mathrm{~h}\right.$ then $\left.60^{\circ} \mathrm{C} \times 48 \mathrm{~h}\right)$. Glass coverslips on embedded blocks were removed following immersion in $49 \%$ (v/v) hydrofluoride for 10-15 min and embedded cells were cut out and sectioned at $70 \mathrm{~nm}(N=3-4$ cells for each group), followed by en bloc staining with $2 \%(\mathrm{w} / \mathrm{v})$ uranyl acetate for $2 \mathrm{~min}$ and Reynold's lead citrate for $2 \mathrm{~min}$. Transmission electron micrographs were acquired using a Philips CM10 electron microscope. To collect images for analysis of neuritic beads, a neurite was identified by morphological features including continuity with a cell body. All of the neuronal images used in the analysis were nonoverlapping and acquired sequentially from the cell body through its processes. For quantification, mitochondria were classified on the basis of their relationship with nearby MTs in longitudinal sections. Mitochondria or mitochondria-like structures that were flanked by MTs on two sides were defined as 'MT-associated', whereas mitochondria with either no adjacent MTs or those with MTs on only one side were defined as 'MT-dissociated'.

Mitochondria/cytosol fractionation and reconstitution assay. Subcellular fractionation was performed using a Mitochondria/Cytosol Fractionation Kit (BioVision), following the manufacturer's protocol. Purified mitochondrial fractions were re-suspended in buffer and total protein determined using the Total Protein Kit (Sigma-Aldrich). For the reconstitution assay, $40 \mu \mathrm{g}$ total protein from the mitochondrial fraction of untreated primary neurons was incubated with $0.5-2.0 \mu \mathrm{g} \mathrm{Ub}, \mathrm{UbB}+1$ or a polyubiquitinated form of $\mathrm{UbB}+1, \mathrm{Ub} 5+1$, in a total volume of $40 \mu \mathrm{l}$ for $20 \mathrm{~min}$ at room temperature. Following brief vortexing and centrifugation at 12000 r.p.m. $\left(4^{\circ} \mathrm{C} 15 \mathrm{~min}\right)$, the mitochondrial pellet and supernatant were each mixed with protein-loading buffer and analyzed by Western blotting for P74 abundance.
Western blotting. Western blotting was performed as described previously. ${ }^{18,19} \beta$-actin, porin and GAPDH were used as loading controls for whole-cell lysates, mitochondrial and cytosolic fractions, respectively. Primary antibodies were used at a concentration of $1: 100$ (kinesin heavy chain, KIF1B), $1: 200$ (Ub, UbB +1, EGFP, p53, p74, GAPDH) or 1:1000 ( $\beta$-actin, porin). Quantification of gel pixel intensity was performed with UN-Scan-It software.

Small Interfering RNA-mediated suppression of UbB +1 . siRNA corresponding to $\mathrm{UbB}+1 \mathrm{mRNA}$ with the dinucleotide deletion was designed with two base overhangs on each strand and prepared using the Ambion siRNA construction kit and the following oligonucleotide templates: sense $5^{\prime}$-AAAGAG GGTATGCAGATCTTCCCTGTCTC-3' and antisense $5^{\prime}$-AAGAAGATCTGCATACC CTCTCCTGTCTC-3' according to the manufacturer's instructions. A construct expressing a hairpin siRNA and a fluorescent reporter under the direction of two different promoters was developed from Clontech pDsRed2-1 and Ambion pSilencer-1.0 U6 vectors as well as published data. Co-transfection of reporter plasmids and $\mathrm{UbB}+1$ siRNA was performed using identical conditions as for plasmid transfections with Lipofectamine 2000. pEGFP-UbB was used as a control for specificity of UbB + 1 RNA interference.

All of the results were confirmed by three or four independent experiments. Statistical analysis was performed using JMP 6 software (SAS Institute, Cary, NC, USA). Cell counts and gel pixel intensity were evaluated by analysis of variance (ANOVA) with post-hoc Fisher's protected least significant difference (PLSD) procedure or Student's t-test.

Acknowledgements. This study was sponsored by NIH AG-26637 to Z.T., an Alzheimer's Association IIRG grant to S.S.S., NIH NS 27501 to D.K.O.D., NS 33213 to M.A.S., and grants from the Jan Dekker Stichting and Dr Ludgardine Bouwman Stichting (04-22), Internationale Stichting Alzheimer's Onderzoek (04507/04830), the Van Leersum Foundation, the Hersenstichting Nederland (13F05.11) and the Hersenstichting Nederland-2005, the Matty Brand Stichting, the van Leersum Foundation (2006) and the Society for Progressive Supranuclear Palsy (440-4) to F.W.V.L.

1. Hershko A, Ciechanover A. The ubiquitin system. Annu Rev Biochem 1998; 67: 425-479.

2. Schwartz AL, Ciechanover A. The ubiquitin-proteasome pathway and pathogenesis of human diseases. Annu Rev Med 1999; 50: 57-74.

3. Jesenberger V, Jentsch S. Deadly encounter: ubiquitin meets apoptosis. Nat Rev Mol Cell Biol 2002; 3: 112-121.

4. Scheper W, Hol EM. Protein quality control in Alzheimer's disease: a fatal saviour. Curr Drug Targets CNS Neurol Disord 2005; 4: 283-292.

5. Fischer DF, De Vos RA, Van Dijk R, De Vrij FM, Proper EA, Sonnemans MA et al. Diseasespecific accumulation of mutant ubiquitin as a marker for proteasomal dysfunction in the brain. FASEB J 2003; 17: 2014-2024.

6. Hol EM, van Leeuwen FW, Fischer DF. The proteasome in Alzheimer's disease and Parkinson's disease: lessons from ubiquitin B+1. Trends Mol Med 2005; 11: 488-495.

7. van Leeuwen FW, de Kleijn DP, van den Hurk HH, Neubauer A, Sonnemans MA, Sluijs JA et al. Frameshift mutants of beta amyloid precursor protein and ubiquitin-B in Alzheimer's and Down's patients. Science 1998; 279: 242-247.

8. Lam YA, Pickart CM, Alban A, Landon M, Jamieson C, Ramage $R$ et al. Inhibition of the ubiquitin-proteasome system in Alzheimer's disease. Proc Natl Acad Sci USA 2000; 97: 9902-9906

9. Lindsten K, de Vrij FM, Verhoef LG, Fischer DF, van Leeuwen FW, Hol EM et al. Mutant ubiquitin found in neurodegenerative disorders is a ubiquitin fusion degradation ubstrate that blocks proteasomal degradation. J Cell Biol 2002; 157: 417-427.

10. De Vrij FM, Sluijs JA, Gregori L, Fischer DF, Hermens WT, Goldgaber D et al. Mutant ubiquitin expressed in Alzheimer's disease causes neuronal death. FASEB J 2001; 15 : 2680-2688.

11. Qian SB, Ott DE, Schubert U, Bennink JR, Yewdell JW. Fusion proteins with $\mathrm{COOH}$ terminal ubiquitin are stable and maintain dual functionality in vivo. J Biol Chem 2002; 277 : 38818-38826.

12. Aridor M, Hannan LA. Traffic jams II: an update of diseases of intracellular transport. Traffic 2002; 3: 781-790.

13. Hollenbeck PJ, Saxton WM. The axonal transport of mitochondria. J Cell Sci 2005; 118: 5411-5419.

14. Goldstein LS, Yang Z. Microtubule-based transport systems in neurons: the roles of kinesins and dyneins. Annu Rev Neurosci 2000; 23: 39-71.

15. Nangaku M, Sato-Yoshitake R, Okada Y, Noda Y, Takemura R, Yamazaki H et al. KIF1B, a novel microtubule plus end-directed monomeric motor protein for transport of mitochondria. Cell 1994; 79: 1209-1220. 
16. Morris RL, Hollenbeck PJ. Axonal transport of mitochondria along microtubules and F-actin in living vertebrate neurons. J Cell Biol 1995; 131: 1315-1326.

17. Hirokawa N, Takemura R. Molecular motors and mechanisms of directional transport in neurons. Nat Rev Neurosci 2005; 6: 201-214

18. Tan Z, Tu W, Schreiber SS. Downregulation of free ubiquitin: a novel mechanism of p53 stabilization and neuronal cell death. Brain Res Mol 2001; 91: 179-188.

19. Tan Z, Qu W, Tu W, Liu W, Baudry M, Schreiber SS. p53 accumulation due to down-regulation of ubiquitin: relevance for neuronal apoptosis. Cell Death Differ 2000; 7 : 675-681.

20. Miller VM, Xia H, Marrs GL, Gouvion CM, Lee G, Davidson BL et al. Allele-specific silencing of dominant disease genes. Proc Natl Acad Sci USA 2003; 100: 7195-7200.

21. Roediger $B$, Armati PJ. Oxidative stress induces axonal beading in cultured human brain tissue. Neurobiol Dis 2003; 13: 222-229.

22. Ivins KJ, Bui ET, Cotman CW. Beta-amyloid induces local neurite degeneration in cultured hippocampal neurons: evidence for neuritic apoptosis. Neurobiol Dis 1998; 5: 365-378.

23. Takeuchi H, Mizuno T, Zhang G, Wang J, Kawanokuchi J, Kuno R et al. Neuritic beading induced by activated microglia is an early feature of neuronal dysfunction toward neurona death by inhibition of mitochondrial respiration and axonal transport. J Biol Chem 2005 280: 10444-10454.

24. Berse M, Bounpheng M, Huang X, Christy B, Pollmann C, Dubiel W. Ubiquitin-dependent degradation of Id 1 and Id3 is mediated by the COP9 signalosome. J Mol Biol 2004; 343 361-370.

25. Hirokawa N. Kinesin and dynein superfamily proteins and the mechanism of organelle transport. Science 1998; 279: 519-526.

26. Martin M, lyadurai SJ, Gassman A, Gindhart Jr JG, Hays TS, Saxton WM. Cytoplasmic dynein, the dynactin complex, and kinesin are interdependent and essential for fast axonal transport. Mol Biol Cell 1999; 10: 3717-3728.

27. Bardag-Gorce F, Riley N, Nguyen V, Montgomery RO, French BA, Li J et al. The mechanism of cytokeratin aggresome formation: the role of mutant ubiquitin $(\mathrm{UBB}+1)$ Exp Mol Pathol 2003; 74: 160-167.
28. Johnston JA, Illing ME, Kopito RR. Cytoplasmic dynein/dynactin mediates the assembly of aggresomes. Cell Motil Cytoskeleton 2002; 53: 26-38.

29. Hope AD, Lashley $T$, Lees AJ, De Silva R. Failure in heat-shock protein expression in response to UBB+1 protein in progressive supranuclear palsy in humans. Neurosci Lett 2004; 359: 94-98.

30. Tsai MY, Morfini G, Szebenyi G, Brady ST. Release of kinesin from vesicles by hsc70 and regulation of fast axonal transport. Mol Biol Cell 2000; 11: 2161-2173.

31. Hirokawa N, Takemura R. Molecular motors in neuronal development, intracellular transport and diseases. Curr Opin Neurobiol 2004; 14: 564-573.

32. Song MS, Saavedra L, De Chaves El. Apoptosis is secondary to non-apoptotic axona degeneration in neurons exposed to Abeta in distal axons. Neurobiol Aging 2005; 27 : 1224-1238.

33. Mandelkow EM, Stamer K, Vogel R, Thies E, Mandelkow E. Clogging of axons by tau, inhibition of axonal traffic and starvation of synapses. Neurobiol Aging 2003; 24 1079-1085.

34. Pigino G, Morfini G, Pelsman A, Mattson MP, Brady ST, Busciglio J. Alzheimer's presenilin 1 mutations impair kinesin-based axonal transport. $J$ Neurosci 2003; 23: 4499-4508.

35. Gunawardena S, Goldstein LS. Disruption of axonal transport and neuronal viability by amyloid precursor protein mutations in Drosophila. Neuron 2001; 32: 389-401.

36. Maki CG, Huibregtse JM, Howley PM. In vivo ubiquitination and proteasome-mediated degradation of p53(1). Cancer Res 1996; 56: 2649-2654.

37. Mihara M, Erster S, Zaika A, Petrenko O, Chittenden T, Pancoska P et al. p53 has a direct apoptogenic role at the mitochondria. Mol Cell 2003; 11: 577-590.

38. Goldbaum O, Vollmer G, Richter-Landsberg C. Proteasome inhibition by MG-132 induces apoptotic cell death and mitochondrial dysfunction in cultured rat brain oligodendrocytes but not in astrocytes. Glia 2006; 53: 891-901.

39. van Leeuwen FW, Fischer DF, Kamel D, Sluijs JA, Sonnemans MA, Benne R et al. Molecular misreading: a new type of transcript mutation expressed during aging. Neurobiol Aging 2000; 21: 879-891.

Supplementary Information accompanies the paper on Cell Death and Differentiation website (http://www.nature.com/cdd) 